\title{
Intensificación de floraciones de dinoflagelados marinos en Uruguay
}

\section{Intensification of marine dinoflagellates blooms in Uruguay}

\author{
Martínez, Ana (1); Méndez, Silvia (1); Fabre, Amelia (2); Ortega, Leonardo (1). \\ (1) Dirección Nacional de Recursos Acuáticos, Ministerio de Ganadería, Agricultura y Pesca (MGAP), Uruguay. \\ (2) Facultad de Ciencias, Universidad de la República (UdelaR), Uruguay.
}

Contacto: anamart30@gmail.com

RECIBIDO: 3/4/2017-APROBADO: 15/6/2017

\begin{abstract}
Resumen
Las pérdidas económicas y problemas de salud ocasionados por las Floraciones Algales Nocivas (FAN) hacen fundamental su comprensión. Mientras continúa el debate sobre si el aumento de los reportes se debe a mayor actividad de monitoreo o a un incremento real en su ocurrencia, los problemas causados por las FAN están creciendo mundialmente. Se espera que las FAN aumenten a causa del cambio climático por un incremento en la temperatura superficial del mar, mayor estratificación, acidificación del océano y eutrofización, principalmente en las zonas costeras. Uruguay no escapa a esa tendencia y en los últimos años ha mostrado un incremento en la intensidad de algunas floraciones (mayor densidad y duración), aunque sin cambios en su frecuencia. Especies que comúnmente producían floraciones en la actualidad lo hacen con mayor abundancia $\left(10^{6}\right.$ cél $\left./ 1\right)$ y duración (entre 3 y 5 semanas). Lo mismo ocurre con algunas especies tóxicas (i.e. Dinophysis cf. acuminata) para las cuales la duración de las vedas en moluscos ha mostrado un incremento, con un máximo de 189 días durante 2015. El aumento de estas floraciones, tanto en el espacio como en el tiempo, podría estar asociado a un aumento de la temperatura y la concentración de nutrientes.

Palabras clave: FAN, fitoplancton marino, mareas rojas, cambio climático.

Abstract

The economic losses and health problems caused by Harmful Algal Blooms (HAB) make their understanding fundamental. While the debate continues over whether increased reporting is due to improved monitoring activity or a real increase, $\mathrm{HAB}$ are expanding worldwide and they are expected to increase as a result of climate change due to increased sea surface temperature, increased stratification, ocean acidification and eutrophication, mainly in coastal areas. Uruguay is not an exception to this trend and in recent years has shown an increase in the intensity of some blooms (higher density and duration), but without changes in its frequency. Species that commonly produced blooms nowadays do so with higher abundance $\left(10^{6}\right.$ cells / l) and duration (between three and five weeks). The same is occurring with toxic species (e.g. Dinophysis cf. acuminata) determining an extension in time of the mollusks bans, with a maximum of 189 days during 2015. The increase in both space and time of these blooms could be associated with an increase in temperature and nutrient concentration.

Keywords: $\mathrm{HAB}$, marine phytoplankton, red tides, climate change.
\end{abstract}

\section{Introducción}

Los ecosistemas acuáticos son sustentados por organismos fotosintéticos que fijan carbono, producen oxígeno y constituyen la base de la cadena trófica. Las floraciones (proliferaciones celulares) son una parte natural del ciclo estacional de los organismos fotosintéticos en los ecosistemas marinos. Ellos son un componente clave en la estructura y dinámica de los océanos y sostienen importantes servicios ecosistémicos. No obstante, algunas floraciones pueden tener efectos negativos y causar daño a humanos u otros organismos y son descritas en su conjunto como Floraciones Algales Nocivas (FAN o HAB por su sigla en inglés), término que incluye una variedad de especies y consecuencias adversas. Las FAN ocurren en todos los ecosistemas acuáticos (dulces, salobres y marinos) y en todas las latitudes (Hallegraeff, 1993).
De los cientos de especies de microalgas descritas, alrededor de 300 están involucradas en eventos nocivos y aproximadamente unas 100, sin vínculo filogenético o fisiológico, producen potentes toxinas naturales que pueden ser dañinas e incluso letales a humanos y animales (Moestrup, et al., 2009 en curso), a menudo vía transferencia de toxinas por la cadena trófica. Otras especies liberan sustancias que no son estrictamente toxinas, pero que causan daño a otros organismos afectando el ecosistema, las pesquerías y las condiciones del agua para uso recreativo (Anderson, et al., 2012).

Por lo tanto, los impactos de las FAN son muy amplios y comprenden efectos agudos y crónicos en la salud humana, pérdidas económicas por productos del mar contaminados, mortalidades de peces en centros de cultivo, usos recreacionales y comerciales de los ambientes marinos costeros como la pesca, colecta de moluscos y turismo costero (Méndez, 
2006). Pueden provocar graves impactos socioeconómicos por cierre de pesquerías de moluscos (Jin, et al., 2008) o un aumento en los costos de monitoreo y manejo (Hoagland, et al., 2002), aunque el cálculo de estas pérdidas es difícil porque depende de la región, la cotización del producto pesquero, el tiempo de clausura de la pesquería, etcétera.

A su vez, ciertas FAN tienen consecuencias nocivas para organismos y ecosistemas, fundamentalmente por alteración de procesos biológicos y fisiológicos (conducta alimenticia, estadíos de vida, estrategias de supervivencia, capacidad reproductiva) de otros organismos o tramas tróficas a través de efectos alelopáticos (Fistarol, et al., 2003; Granéli y Hansen, 2006), llevando a la degradación de los servicios ecosistémicos y pérdida de valores pasivos (no de mercado) asignados por el hombre para especies protegidas y ecosistemas (Berdalet, et al., 2015).

Asimismo, la senescencia de grandes floraciones y su subsiguiente degradación por bacterias reduce la concentración de oxígeno y causa hipoxia, particularmente en las aguas de fondo, pudiendo causar mortalidades de la fauna bentónica. Se estima que más del $50 \%$ de los eventos inusuales de mortandades de fauna marina (tortugas, delfines, ballenas, aves) pueden deberse a biotoxinas marinas (Scholin, et al., 2000). Sumado a esto algunas FAN tienen asociada una espuma superficial de apariencia desagradable, mal olor e incluso aerosoles tóxicos que causan irritación de las vías respiratorias, que puede disuadir a turistas del uso recreativo de estas áreas (Scatasta, et al., 2003; Backer, et al., 2005; Gorbi, et al., 2013). Aunque existe considerable conocimiento de los efectos en la salud humana causados por las biotoxinas, muchos de los mecanismos toxicológicos aún se desconocen y poco se sabe de los efectos crónicos de estas toxinas, tanto de la exposición aguda como de la exposición crónica a bajos niveles durante períodos prolongados (Berdalet, et al., 2015).

El fenómeno de las FAN data de un par de siglos atrás; el primer registro de caso fatal de envenenamiento por consumo de moluscos contaminados con toxinas de dinoflagelados se registró en 1793, cuando el capitán Vancouver y su tripulación arribaron en British Columbia, en un área denominada hoy Poison Cove, donde para las tribus locales era tabú comer mariscos cuando el agua estaba fosforescente debido a floraciones de dinoflagelados (Dale y Yentsch, 1978). Si bien las floraciones son un fenómeno natural, se reconoce que pueden verse favorecidas por presiones antropogénicas en las áreas costeras, de hecho se ha registrado un incremento en las últimas décadas a escala global (Hallegraeff, 1993; Anderson, et al., 2012). Debido al incremento de la presión humana en las zonas costeras, que genera cambios en la circulación de agua por puertos y playas artificiales, dispersión de especies por agua de lastre y enriquecimiento con nutrientes (Anderson, et al., 2002; Davidson, et al., 2014) y junto con el cambio climático, se pueden registrar FAN en áreas donde no ocurrían y donde no habían sido reportadas previamente (Hallegraeff, 2010; Trainer, et al., 2013). Específicamente, pueden ocurrir cambios en la frecuencia, intensidad y extensión geográfica de FAN, pero las posibles respuestas son probablemente especie-específica, dada la diversidad de toxicidad, fisiología, biología y ecología de los organismos involucrados. A su vez, la detección de nuevas toxinas y los organismos causativos representan nuevos desafíos para los procedimientos de monitoreo y manejo de los eventos nocivos (Turner, et al., 2015).

El objetivo de este trabajo es describir el incremento que se ha registrado en Uruguay en la intensidad de las floraciones de algunas especies y discutir su probable asociación con las tendencias climáticas regionales y la eutrofización.

\section{Material y Métodos}

Los datos provienen del programa de monitoreo de FAN que es llevado a cabo por la Dirección Nacional de Recursos Acuáticos (DINARA) desde el año 1980. En este programa se obtienen muestras semanales en puntos fijos de la costa oceánica uruguaya (departamentos de Maldonado y Rocha) donde es frecuente la extracción de moluscos bivalvos ( $\mathrm{Fi}$ gura 1). En cada punto se obtienen muestras de agua para estudio fitoplanctónico y muestras de moluscos bivalvos para extracción y análisis de toxinas mediante bioensayo en ratones. Las muestras para análisis cualitativo de fitoplancton son extraídas con red de $25 \mu \mathrm{m}$ y son fijadas con formaldehído neutro al $4 \%$. Las muestras para análisis cuantitativo son fijadas con Lugol ácido (Throndsen, 1978) y, siguiendo el método Utermöhl (1958), son analizadas al microscopio invertido (Olympus IM y Leitz Labovert FS).

Para este trabajo se analizaron datos de abundancia de algunas especies, se consideraron solo los máximos anuales correspondientes a floraciones, desde la década de 1990 hasta la fecha. Se eligieron especies que suelen hacer floraciones conspicuas, provocando coloración del agua, pero que no son tóxicas (Akashiwo sanguinea, Alexandrium fraterculus, Heterocapsa triquetra) y una especie tóxica (Dinophysis acuminata) cuyas floraciones son de mucho menor abundancia pero suele generar períodos de veda de actividades extractivas de moluscos. Dinophysis acuminata es considerada un complejo de especies, de las cuales en Uruguay se ha identificado recientemente también Dinophysis ovum (Méndez, et al., 2016a). A los efectos de esta publicación se consideran ambas especies de Dinophysis como Dinophysis cf. acuminata para poder comparar los datos actuales (en los que están diferenciadas ambas especies) con datos anteriores en los que se la consideraba una sola especie. Las abundancias fueron logaritmizadas para disminuir su rango de variación. También se utilizó la suma anual de las abundancias máximas de las cuatro especies durante las floraciones. Los datos de anomalías de temperatura superficial del mar (SSTA) para la región (Ortega, et al., 2016) así como los datos de anomalías en las precipitaciones fueron obtenidos de la página de servicios climáticos de libre acceso del International Research Institute (IRIDL, s.d. a; IRIDL, s.d. b; Reynolds, et al., 2002 y Janowiak, et al., 1999., respectivamente). Se calculó la suma acumulada de las anomalías para detectar cambios en el régimen climático, los cuales se evidencian por puntos de inflexión en la curva de la suma acumulada (Fiedler, 2002).

\section{Resultados y Discusión}

Para todas las especies analizadas se observó un aumento en la densidad de las floraciones, principalmente en la última década, y al considerar la abundancia máxima acumulada de todas estas especies hay un notorio aumento a partir del año 2000 (Figura 2).

Si bien la frecuencia de las floraciones no ha variado y siguen ocurriendo en las mismas épocas del año, han aumentado su abundancia en varios órdenes de magnitud, llegando muchas de ellas a $10^{7}$ células/ litro. Este aumento es más 


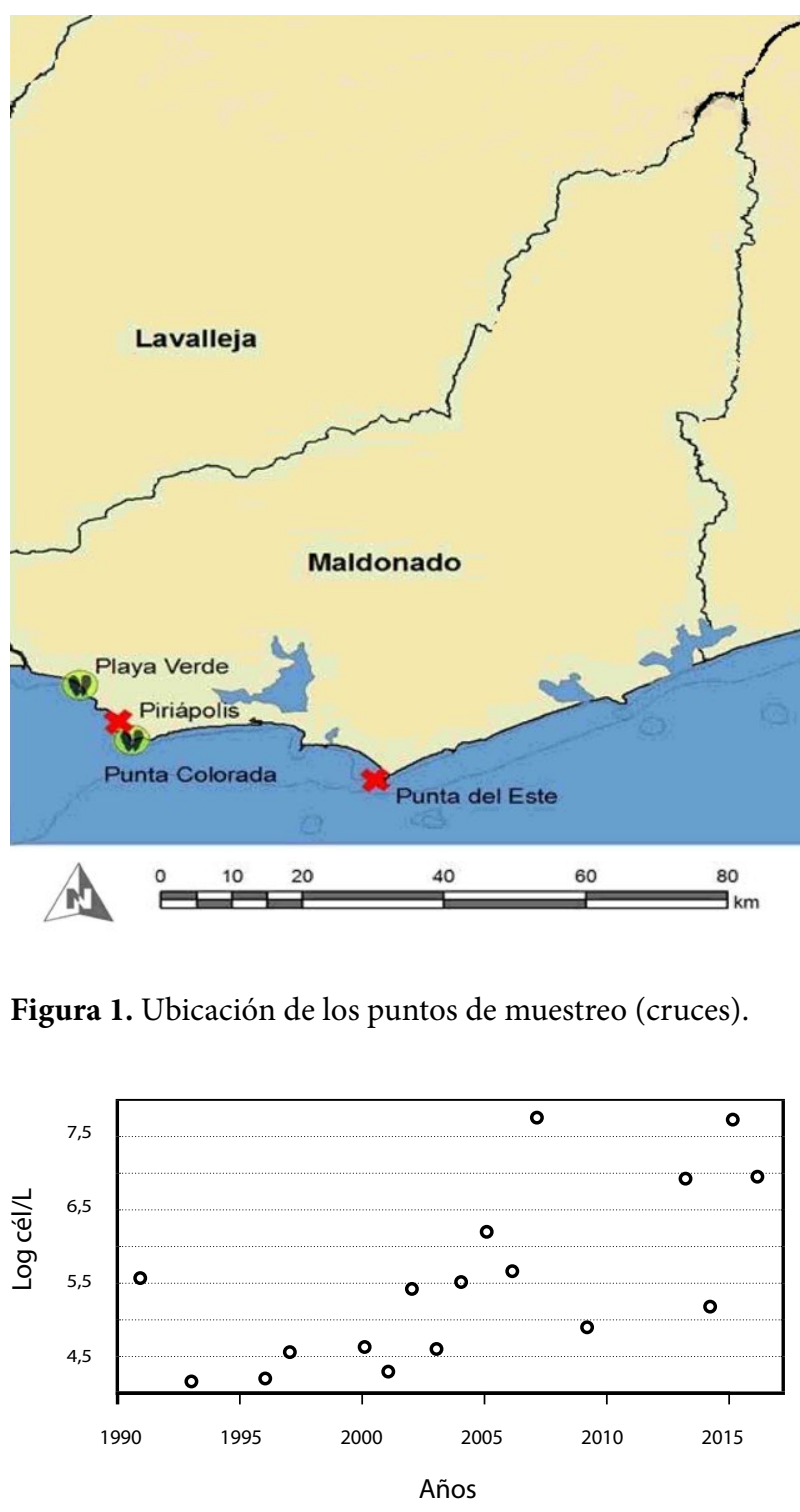

Figura 2. Abundancia celular máxima anual acumulada para todas las especies consideradas Akashiwo sanguinea, Alexandrium fraterculus, Dinophysis acuminata y

Heterocapsa triquetra registradas en la costa de Maldonado y Rocha desde 1991 hasta 2016.

marcado aún en las especies con preferencias cálidas, como lo son D. cf. acuminata y A. fraterculus (Figura 3). En particular, estas dos especies presentaron una floración extrema en el año 2015, más notoria para Dinophysis, en la cual la mayoría de sus floraciones son de $10^{3}$ cél/l y en 2015 alcanzó $1.56 \mathrm{X}$ $10^{5}$ cél/l (Méndez, et al., 2016b). A. fraterculus alcanzó una abundancia record de $4.77 \times 10^{7}$ cél/l (Martínez, et al., 2016). El año 2015 fue particularmente seco y cálido, lo cual se evidenció en anomalías negativas de precipitación en la cuenca del río Uruguay a partir de febrero (Figura 4 A). Esto, a su vez, resulta en una menor descarga de agua dulce y permite que las aguas cálidas provenientes del norte se acerquen a la costa (Ortega y Martínez, 2007), provocando una fuerte anomalía térmica positiva (Figura $4 \mathrm{~B}$ ). Por su parte, el sur de Brasil y la costa oceánica de Uruguay están catalogados como «hotspots» del calentamiento marino costero (Hobday, et al., 2016), por lo cual se espera que la tendencia siga en aumento.

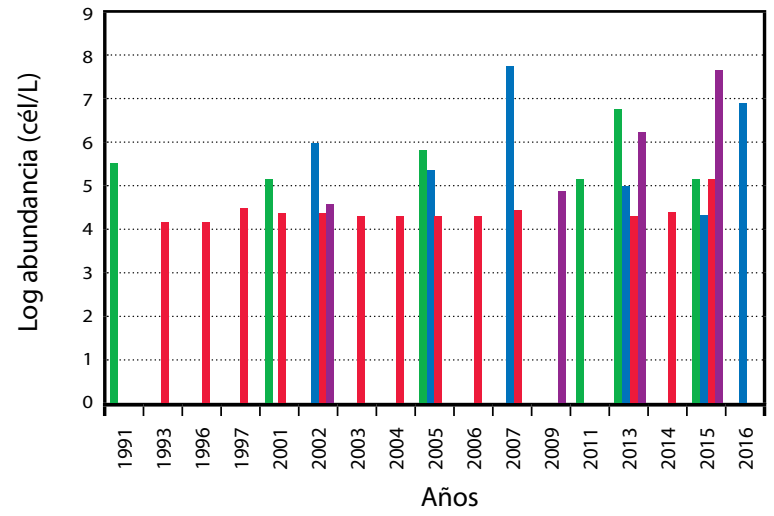

Figura 3. Abundancia celular máxima durante las floraciones de Akashiwo sanguinea (verde), Alexandrium fraterculus (violeta), Dinophysis acuminata (rojo) y Heterocapsa triquetra (azul) registradas en la costa de Maldonado y Rocha desde 1991 hasta 2016.
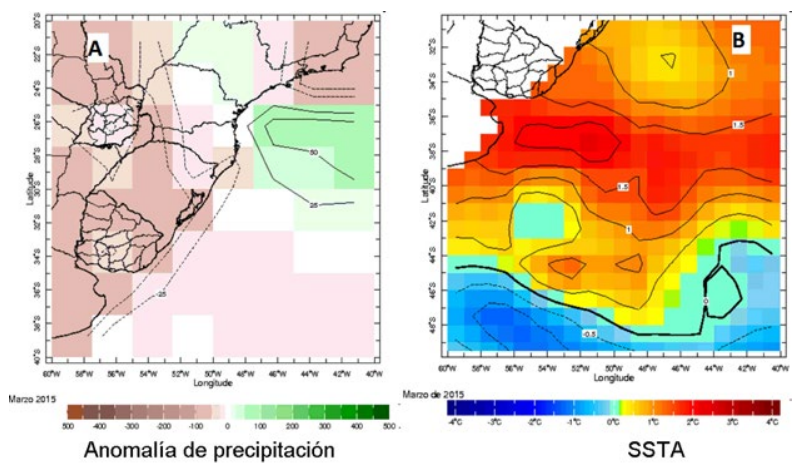

Figura 4. A) Anomalías de precipitaciones ( $\mathrm{mm} / \mathrm{mes})$; B) Anomalías de temperatura superficial del mar (SSTA) $\left({ }^{\circ} \mathrm{C}\right)$ para marzo de 2015. 


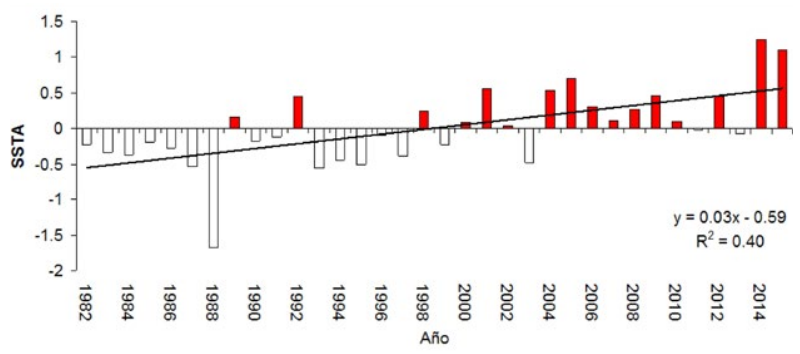

Figura 5. Promedios anuales de anomalías de la temperatura superficial del mar estandarizadas (SSTA, por su sigla en inglés) donde se resaltan las anomalías positivas en rojo y su predominio a partir de 1997. Se incluye la tendencia lineal para mostrar el incremento de temperatura, la SSTA no lleva unidades pues está estandarizada.

El análisis temporal de las anomalías de la temperatura superficial del mar muestra un predominio de anomalías térmicas negativas antes de 1997 y una tendencia hacia un predominio de anomalías térmicas positivas a partir de 1997, más marcadas aún a partir del año 2013 (Figura 5).

Asimismo, se ha documentado un aumento significativo de la temperatura del mar en la costa uruguaya, asociado a la migración del frente de aguas cálidas hacia el sur con un desplazamiento promedio estimado de $9 \mathrm{~km} /$ año (Ortega, et al., 2016). Estas evidencias confirman una tendencia hacia una mayor presencia de aguas más cálidas en la región, lo cual favorecería a las especies de preferencias cálidas.

La tendencia al aumento de floraciones está presuntamente vinculada al cambio climático. A nivel mundial están ocurriendo floraciones sin precedentes (Campbell, et al., 2010; Koening, et al., 2014; Dias, et al., 2015; Trainer, et al., 2016; Hallegraeff, et al., 2016) que podrían estar vinculadas a la presencia de aguas más cálidas (Moore, et al., 2008; Hallegraeff, 2010; Gowen, et al., 2012). Dentro de los posibles impactos del cambio climático se consideran: 1) el cambio en el rango de distribución tanto de especies de aguas cálidas como frías; 2) los cambios de especie específicos en la abundancia y toxicidad; 3) los cambios en la ventana temporal de crecimiento estacional, y 4) los efectos en otros componentes de las cadenas tróficas marinas (predadores, competidores y parásitos) que podrían modular el impacto en la ocurrencia, magnitud y duración de las FAN (Hays, et al., 2005; Moore, et al., 2008; Hallegraeff, 2010; Anderson, et al., 2012; Wells, et al., 2015). En Uruguay ya se están observando los numerales 1 y 2.

La suma acumulada de las anomalías de temperatura superficial del mar registra un cambio de régimen climático en la región a partir de 1997, pasando de régimen frío a uno cálido (Figura 6). Este fenómeno climático trajo aparejada una alteración en la comunidad de fitoplancton con una predominancia de especies de aguas cálidas (e.g A.fratreculus y D.cf. acuminata) y una disminución en la frecuencia de ocurrencia de especies de aguas frías (e.g. A. tamarense). Según Hallegraeff (2010), algunas especies pueden beneficiarse por el cambio climático y, por lo tanto, tener impactos más severos, mientras que otras pueden disminuir sus impactos; en este sentido se prevén cambios en la composición, abundancia y temporalidad de las floraciones. Un cambio en la compo-

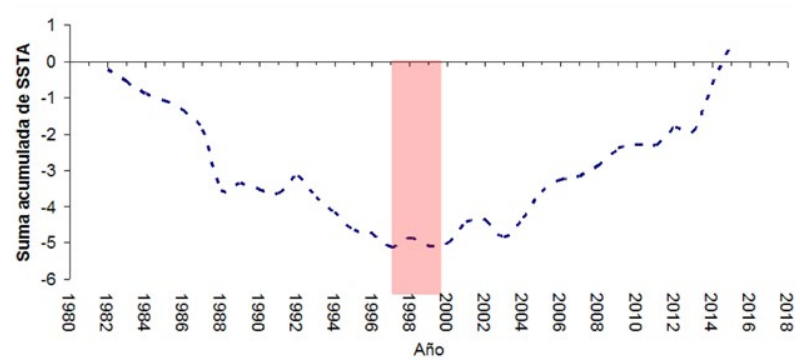

Figura 6. Suma acumulada de anomalías de temperatura superficial del mar (SSTA, por su sigla en inglés) a lo largo del tiempo. Se señala con una barra los años en que se registró el cambio de régimen.

sición de especies de fitoplancton a partir de 1998 también fue registrado por Cloern y Dufford (2005) para la bahía de San Francisco y fue atribuido a un cambio en el ciclo de la Pacific Decadal Oscilation. En Uruguay en los últimos años han sido registradas nuevas especies potencialmente tóxicas (Méndez, et al., 2010).

Las floraciones están aumentando, además, su tiempo de duración; muchas de ellas se mantienen en altísimas densidades por períodos superiores a 4-5 semanas, cuando anteriormente las altas densidades se extendían a un máximo de 3 semanas. El sostener estas altas biomasas por un período prolongado de tiempo requiere de un aporte externo de nutrientes (Heisler, et al., 2008). Este hecho podría deberse a una mayor disponibilidad de nutrientes debido a eutrofización de la zona costera. Si bien la información acerca de la concentración de nutrientes en la costa uruguaya es prácticamente nula, se compararon los escasos datos con los que se cuenta para la costa de Rocha (Arachania y Barra del Chuy) con datos de bibliografía que consideran una serie temporal de 1992 a 2007 (Odebrecht, et al., 2010). La concentración de nutrientes ha aumentado sensiblemente $\left(\mathrm{NO}_{3}+\mathrm{NO}_{2}\right.$ promedio $=35,8 \mu \mathrm{M}$; $\mathrm{PO}_{4}$ promedio $\left.=24,9 \mu \mathrm{M}\right)$ respecto a lo hallado por Odebrecht et al. (2010) $\left(\mathrm{NO}_{3}+\mathrm{NO}_{2}\right.$ máximo $=19,4 \mu \mathrm{M}$ y $\mathrm{PO}_{4}$ máximo= $10,6 \mu \mathrm{M})$, principalmente en Arachania, donde generalmente ocurren las mayores floraciones.

Una hipótesis es que la eutrofización costera está potenciando las floraciones. La costa atlántica uruguaya está siendo cada vez más urbanizada y, en la mayoría de los casos, sin sistema séptico de saneamiento. $\mathrm{El} \mathrm{NO}_{3}$ proveniente de la contaminación del agua subterránea por los sistemas sépticos suele ser la principal fuente de N (Anderson, et al., 2008). Por otro lado, Uruguay ha intensificado significativamente sus usos de suelo en las últimas décadas (principalmente forestación y soja) (Achkar, et al., 2011). En particular, la agricultura intensiva aporta 26 veces más nitrógeno que la forestación y principalmente en forma de nitrato, el cual es más fácilmente utilizable por el fitoplancton (de Wit, et al., 2005). El flujo de fósforo a los océanos se ha incrementado tres veces comparado con los niveles preindustrial, preagricultura, mientras que el flujo de nitrógeno se ha incrementado más de 10 veces en algunos sitios, concomitantemente con un aumento de las FAN en esas áreas, tanto en número 
como en dimensión (Anderson, et al., 2002, 2012). Varias investigaciones han evaluado la posible asociación de la expansión de las FAN con la eutrofización (Anderson, et al., 2002, 2008; Glibert, et al., 2008; Heisler, et al., 2008). En algunos casos el vínculo es claro mientras que en otros es más sutil y complejo o incluso inexistente (Anderson, et al., 2002, 2008). El rango de respuestas fisiológicas de diferentes especies de fitoplancton, la composición relativa de los nutrientes (relación N:P e inorgánicos vs orgánicos) (Anderson, et al., 2002; Heisler, et al., 2008) y las interacciones con otros factores como el pastoreo son importantes en el proceso de modular las respuestas de las FAN a la eutrofización (Heisler, et al., 2008; Anderson, et al., 2012).

A su vez, en el caso de especies tóxicas, como $D$. c $f$. acuminata, está aumentando la duración de los períodos de veda causadas por ella, con un máximo de 189 días de veda durante el año 2015, coincidente con la floración de mayor magnitud registrada hasta el momento (1.56 X $10^{5}$ cél/l). Este aumento significativo en el número de días de duración de las vedas debidas a Dinophysis se correlaciona positivamente con las anomalías de temperatura superficial del mar (Fabre, et al., 2016).

\section{Conclusiones}

Es importante reconocer que no hay una forma realista de prevenir la ocurrencia de las FAN, ya que resultan de una compleja interacción entre factores físicos, químicos y biológicos que operan a diferentes escalas espaciotemporales. Las FAN son un fenómeno natural que ha ocurrido históricamente. Constituyen un problema a escala global, pero la idea de que su ocurrencia pueda prevenirse no es una opción viable por el momento. Los programas de monitoreo de las FAN y sus toxinas, implementados en diferentes lugares del mundo, han reducido claramente los envenenamientos en humanos. Las herramientas disponibles para evaluar este desafío global incluyen el mantenimiento de investigación científica intensiva, multidisciplinaria y colaborativa y el fortalecimiento de la coordinación con actores, políticos y público en general. No existen observaciones directas de microalgas en aguas costeras marinas en escalas temporales suficientemente largas para evaluar el impacto del cambio climático y discriminar su rol de otros forzantes antropogénicos (e.g. carga de nutrientes). Esto dificulta establecer cuándo las tendencias observadas son tendencias globales o locales (Davidson, et al., 2012). Por esto es tan importante la continuación de este monitoreo a largo plazo, en el cual ya comienzan a registrarse ciertas tendencias al aumento en la intensidad de estos eventos.

\section{Referencias}

Achkar, M., Domínguez, A., Díaz, I. y Pesche, F., 2011. La intensificación del uso agrícola del suelo en el litoral oeste del Uruguay en la última década. En: Pampa, 07, pp.143-157.

Anderson, DM., Glibert, PM. y Burkholder, JM., 2002. Harmful algal blooms and eutrophication: nutrient sources, composition, and consequences. En: Estuaries, 25, pp.704-726.

Anderson, DM., Burkholder, JM., Cochlan, WP., Glibert, PM., Gobler, CJ., Heil, C., Kudela, RM., Parsons, M., Rensel, J., Townsend, D., Trainer, VL. y Vargo, G., 2008. Harmful algal blooms and eutrophication: examining linkages from selected coastal regions of the United States. En: Harmful Algae, 8, pp.39-53.

Anderson, DM., Cembella, AD. y Hallegraeff, GM., 2012. Progress in understanding harmful algal blooms: paradigm shifts and new technologies for research, monitoring, and management. En: Annual Review of Marine Science, 4, pp.143-176.

Backer, LC., Fleming, LE., Rowan, A., Cheng, YS, Benson, J., Pierce, RH., Zaias, J., Bean, J., Bossart, GD., Johnson, D., Quimbo, R. y Baden, DG., 2003. Recreational exposure to aerosolized brevetoxins during Florida red tide events. En: Harmful Algae, 2, pp.19-28.

Berdalet, E., Fleming, N., Gowen, R., Davidson, K., Hess, P., Backet, L., Moore, S., Hoagland, P. y Enevoldsen, H., 2015. Marine harmful algal blooms, human health and wellbeing: challenges and opportunities in the $21 \mathrm{st}$ century. En: Journal of Marine Biological Association of the United Kingdom, 96(1), pp.61-91.

Campbell, L., Olson, R., Sosik, H., Abraham, A., Henrichs, D., Hyatt, C. y Buskey, E., 2010. First harmful Dinophysis (Dinophyceae, Dinophysiales) bloom un the US is revealed by automated imaging flow cytometry. En: Journal of Phycology, 46, pp.66-75.

Cloern, J. y Dufford, R., 2005. Phytoplankton community ecology: principles applied in San Francisco Bay. En: Marine Ecology Progress Series, 285, pp.11-28.

Dale, B. y Yentsch, HCM., 1978. Red tide and paralytic shellfish poisoning. En: Oceanus, 21, pp.41-49.

Davidson, K., Gowen, RJ., Tett, P., Bresnan, E., Harrison, PJ., McKinney, A., Milligan, S., Mills, DK., Silke, J. y Crooks, AM., 2012. Harmful algal blooms: how strong is the evidence that nutrient ratios and forms influence their occurrence? En: Estuarine Coastal and Shelf Science, 115, pp.399-413.

Davidson, K., Gowen, RJ., Harrison, PJ., Fleming, L., Hoagland, P. y Moschonas, G., 2014. Anthropogenic nutrients and harmful algae in coastal waters. En: Journal of Environmental Management, 146, pp.206-216.

de Witt, R., Leibreichb, J., Vernierb, F., Delmasb, F., Beuffeb, H., Maisonb, Ph., Chossatb, JC., Laplace-Treytureb, C., Laplanab, R., Clavéb, V., Torreb, M., Aubyc, I., Trutc, G., Maurerc, D. y Capdevilled, P., 2005. Relationship between land-use in the agro-forestry system of les Landes, nitrogen loading to and risk of macro-algal blooming in the Bassin d'Arcachon coastal lagoon (SW France). En: Estuarine Coastal and Shelf Science, 62(3), pp.453-465.

Dias, P., Muñoz, J., Huisman, J. y Mc Donald, J., 2015. Biosecurity monitoring of Harmful Algal Bloom (HAB) species in Western Australian waters: first confirmed record of Alexandrium catenella (Dinophyceae). En: BioInvasions Records, 4(4), pp.233-241.

Fabre, A., Ortega, L., Méndez, S. y Martínez, A., 2016. Bivalve mollusks bans are associated with climate variability in Uruguay. En: ISSHA. $17^{\text {th }}$ International Conference of Harmful Algae. Florinaópolis, Brasil (9-14 de octubre de 2016). Florianópolis: ISSHA

Fiedler, P., 2002. Environmental change in the eastern tropical Pacific Ocean: review of ENSO and decadal variability. En: Marine Ecology Progress Series, 244, pp.265-283.

Fistarol, GO., Legrand, C. y Granéli, E., 2003. Allelopathic effect of Prymnesium parvum on a natural plankton community. En: Marine Ecology Progress Series, 255, pp.115-125. 
Glibert, PM., Mayorga, E. y Seitzinger, S., 2008. Prorocentrum minimum tracks anthropogenic nitrogen and phosphorus inputs on a global basis: application of spatially explicit nutrient export models. En: Harmful Algae, 8, pp.33-38

Gorbi, S. Avio, G.C., Benedetti, M., Totti, C., Accoroni, S., Pichierri, S., Bacchiocchi, S., Orletti, R., Graziosi, T. Regoli, F, 2013. Effects of harmful dinoflagellate Ostreopsis cf ovata exposure on immunological, histological and oxidative responses of mussels Mytilus galloprovincialis. En: Fish \& Shellfish Immunology, 35, pp.941-950.

Gowen, RJ., Tett, P., Bresnan, E., Davidson, K., McKinney, A., Harrison, PJ., Milligan, S., Mills, DK., Silke, J. y Crooks, AM., 2012. Anthropogenic nutrient enrichment and blooms of harmful microalgae. En: Oceanography and Marine Biology: An Annual Review, 50, pp.65-126.

Granéli, E. y Hansen, PJ., 2006. Allelopathy in harmful algae: a mechanism to compete for resources?. En: Granaeli, E. y Turner, JT., eds. Ecology of harmful algae. Berlín: Springer Verlag. pp.189-201.

Hallegraeff, GM., 1993. A review of harmful algal blooms and their apparent global increase. En: Phycologia, 32, pp.79-99.

Hallegraeff, GM., 2010. Ocean climate change, phytoplankton community responses, and harmful algal blooms: a formidable predictive challenge. En: Journal of Phycology, 46, pp.220-253.

Hallegraeff, GM., Bolch, C., Dorantes-Aranda, JJ., Murray, S., Turnbull, A., Ugalde, S. y Wilson, K., 2016. Unprecedented Alexandrium blooms in a previously low biotoxin risk area of Tasmania, Australia. En: ISSHA. 17th International Conference of Harmful Algae. Florinaópolis, Brasil (9-14 de octubre de 2016). Florianópolis: ISSHA

Hays, GC., Richardson, AJ. y Robinson, C., 2005. Climate change and marine plankton. En: Trends in Ecology and Evolution, 20, pp.337-344.

Heisler, J., Gilbert, PM., Burkholder, JM., Anderson, DM., Cochlan, W., Dennison, W., Dortch, Q., Gobler, C., Heil, CA., Humphries, E., Lewitus, A., Magnien, R., Marshall, HG., Sellner, K., Stockwell, DA., Stoecker, D. y Suddlenson, M., 2008. Eutrophication and harmful algal blooms: A scientific consensus. En: Harmful Algae, 8, pp.3-13.

Hoagland, P., Anderson, DM., Kaoru, Y. y White, AW., 2002. The economic effects of harmful algal blooms in the United States: estimates, assessment issues, and information needs. En: Estuaries, 25, pp.677-695.

Hobday, AJ., Cochrane, K., Downey-Breedt, N., Howard, J., Aswani, S., Byfield, V., Duggan, G., Duna, E., Dutra, LXC., Frusher, SD., Fulton, EA., Gammage, L., Gasalla, MA., Griffiths, C., Guissamulo, A., Haward, M., Jarre, A., Jennings, SM., Jordan, T., Joyner, J., Ramani, NK., Shanmugasundaram, SLP., Malherbe, W., Cisneros, K., Paytan, A., Pecl, G., Plaga' nyi E., Popova, EE., Razafindrainibe, H., Roberts, M., Rohit, P., Sainulabdeen, SS., Sauer, W., Valappil, ST., Zacharia, PU. y van Putten I., 2016. Planning adaptation to climate change in fast-warming marine regions with seafooddependent coastal communities. En: Review Fish Biology Fisheries, 26, pp.249-264.

IRIDL, [s.d.]a. Sea surface temperature anomaly data [En línea]. Columbia: IRIDL. [Consulta marzo de 2017]. Disponible en: http://iridl.ldeo.columbia.edu/ SOURCES/.NOAA/.NCEP/.EMC/.CMB/.GLOBAL/. Reyn_SmithOIv2/.monthly/.ssta/
IRIDL, [s.d.]b. Climate anomaly monitoring system-outgoing longwave radiation precipitation index [En línea]. Columbia: IRIDL. [Consulta marzo de 2017]. Disponible en:http:// iridl.ldeo.columbia.edu/SOURCES/.NOAA/.NCEP/. CPC/.CAMS_OPI/

Janowiak, JE. y Xie, P., 1999: CAMS_OPI: A global satelliterain gauge merged product for real-time precipitation monitoring applications. En: Journal of Climate, 12, pp.3335-3342.

Jin, D., Thunberg, E. y Hoagland, P., 2008. Economic impact of the 2005 red tide event on commercial shellfish fisheries in New England. En: Ocean and Coastal Management, 51, pp.420-429.

Koening, ML., Flores Montes, MJ., Ezkinazi, LE. y Tiburcio, ASXS., 2014. New record of Akashiwo sanguínea (Dinophyta) in the tropical estuarine waters of Northeastern Brazil (Western Atlantic). En: Brazilian Journal of Biology, 74(1), pp.191-198.

Méndez, S., 2006. Impacto de las floraciones algales nocivas en Uruguay: origen, dispersión, monitoreo, control y mitigación. En: Menafra, R., Rodríguez-Gallego, L., Scarabino, F. y Conde, D., eds., 2006. Bases para la conservación y el manejo de la costa uruguaya. Montevideo: Vida Silvestre Uruguay.

Méndez, SM., Akselman, R. y Tomas, CR., 2010. First report of Heterosigma akashiwo, Fibrocapsa japonica and Chattonella marina var antique in Uruguay. En: ICHA. $14^{\text {th }}$ ICHA Conference Proceedings. Creta, Grecia (1-5 de noviembre de 2010). Creta: ICHA

Méndez, SM., Rodríguez, F., Reguera, B., Franco, JM., Riobó, P. y Fabre, A., 2016a. Characterization of a Dinophysis of the acuminata complex causing an excepcional DSP event in Uruguay 2015. En: ISSHA. $17^{\text {th }}$ International Conference of Harmful Algae. Florinaópolis, Brasil (9-14 de octubre de 2016). Florianópolis: ISSHA

Méndez, SM., Martínez, A. y Fabre, A., 2016b. Extreme abundant bloom of Dinophysis of the acuminata complex associated to warm marine waters in Uruguay. En: ISSHA. $17^{\text {th }}$ International Conference of Harmful Algae. Florinaópolis, Brasil (9-14 de octubre de 2016). Florianópolis: ISSHA

Martínez, A., Méndez, SM. y Fabre, A., 2016. First record of bioluminiscence of Alexandrium fraterculus (dinoflagellate), in the Uruguayan coast, South Western Atlantic Ocean. En: Pan-American Journal of Aquatic Science, 11(4), pp.356-360.

Moestrup, Ø., Akselman, R., Cronberg, G., Elbraechter, M., Fraga, S., Halim, Y., Hansen, G., Hoppenrath, M., Larsen, J., Lundholm, N., Nguyen, LN. y Zingone, A., eds., 2009-. IOC-UNESCO taxonomic reference list of harmful micro algae [En línea]. [s.l.]: UNESCO. [Consulta 10 de marzo de 2017] Disponible en: http://www.marinespecies.org/hab

Moore, SK., Trainer, VL., Mantua, NJ., Parker, MS., Laws, EA., Backer, LC. y Fleming, LE., 2008. Impacts of climate variability and future climate change on harmful algal blooms and human health. En: Environmental Health, 7(2), pp.1-12.

Odebrecht, C., Bergesh, M., Rörig, LR. y Abreu, PC., 2010. Phytoplankton Interanual Variability at Cassino Beach, Southern Brazil (1992-2007), with Emphasis on the Surf Zone Diatom Asterionellopsis glacialis. En: Estuaries and Coasts, 33, pp.570-583.

Ortega, L. y Martínez, A., 2007. Multiannual and Seasonal Variability of Water Masses and Fronts Over the Uruguayan Shelf. En: Journal of Coastal Research, 23(3), pp.618-629. 
Ortega, L., Celentano, E., Delgado, E y Defeo, O., 2016. Climate change influences on abundance, individual size and body abnormalities in a sandy beach clam. En: Marine Ecology Progress Series, 545, pp.203-213.

Reynolds, RW., Rayner, NA., Smith, TM., Stokes, DC. y Wang, W., 2002. An improved in situ and satellite SST analysis for climate. En: Journal of Climate, 15, pp.1609-1625.

Scatasta S., Stolte W., Graneeli E. y van Ierland E.. 2003. The socio economic impact of harmful algal blooms in european marine waters and description of future risks. [s.l.]: Unión Europea.

Scholin, CA., Gulland, F., Doucette, GJ., Benson, S., Busman, M., Chavez, FP., Cordaro, J., DeLong, R., De Vogelaere, A., Harvey, J., Haulena, M., Lefebvre, K., Lipscomb, T. y Van Dolah, FM., 2000. Mortality of sea lions along the central California coast linked to a toxic diatom bloom. En: Nature, 403, pp.80-84.

Trainer, VL., Moore, L., Bill, BD., Adams, NG., Harrington, N., Borchert, J., Silva, DAM. y Eberhart, BTL., 2013. Diarrhetic shellfish toxins and other lipophilic toxins of human health concern in Washington State. En: Marine Drugs, 11, pp.1815-1835.
Trainer, VL., McCabe, RM., Hickey, B., Kudela, RM., Lefebvre, K., Adams, NG., Bill, BD., Gulland, FMD., Thomson, R. y Cochlan, W., 2016. En: ISSHA. 17th International Conference of Harmful Algae. Florinaópolis, Brasil (9-14 de octubre de 2016). Florianópolis: ISSHA

Throndsen, J., 1978. Preservation and storage. En: Sournia, A., ed., 1978. Phytoplankton manual. Paris: UNESCO. (UNESCO Monographic Oceanographic Method, 6). pp.69-74.

Turner, A., Higgins, C., Veszelovski, A., Payne, D., Davidson, K., Hungerford, J. y Higman, W., 2015. Monitoring of new or emerging marine biotoxins in UK waters: brevetoxins. En: Marine Drugs, 13, pp.1224-1254.

Utermöhl, H., 1958. Zur vervollkomnung der quantitativen phytoplankton metodik. En: Mitteilung Internationale Vereinigung fuer Limnologie, 9, pp.1-38.

Wells, ML., Trainer, VL., Smayda, T., Karlson, B., Trock, CG., Kudela, RM., Ishikawa, A., Bernard, S., Wulff, A., Anderson, D. y Cochlan, W., 2015. Harmful algal blooms and climate change: Learning from the past and present to forecast the future. En: Harmful Algae, 49, pp.68-93. 